\title{
Steady flow of a Reiner-Rivlin fluid between rotating plates
}

\author{
Lawrence K. Forbes ${ }^{\mathrm{a})}$ \\ School of Mathematics and Physics, University of Tasmania, P.O. Box 37, Hobart 7001, Tasmania, Australia
}

(Received 27 August 2018; accepted 10 October 2018; published online 25 October 2018)

\begin{abstract}
This paper considers a von Kármán type axisymmetric flow between parallel plates, in which the top plate rotates and the bottom one is stationary. Between the plates is a weakly non-Newtonian fluid of Reiner-Rivlin type. A highly accurate spectral method is presented for solving the steady problem, and Newton's method is used to find the Fourier coefficients and an eigenvalue. Multiple solutions are found, of which one is clearly of Batchelor type and another is clearly of Stewartson type, and these persist in the non-Newtonian regime. Such flows may be of practical use in viscometry, in which the coefficient of the non-Newtonian viscous term might be measured. Published by AIP Publishing. https://doi.org/10.1063/1.5053833
\end{abstract}

\section{INTRODUCTION}

The original motivation for this work came from a model of the transition of viscous fluid flow from laminar to turbulent, proposed by Forbes ${ }^{1,2}$ and developed further by Forbes and Brideson ${ }^{3}$ for swirling flow in a round pipe. In simple geometries, such as Couette flow between moving plates or Poiseuille flow in a pipe, the Navier-Stokes equations of viscous flow admit simple closed-form solutions, and these are believed to be stable to infinitesimal disturbances for all Reynolds numbers $R_{e}$ (see Ref. 4). Forbes, ${ }^{1}$ however, argued that the linear Newtonian relationship between stress and strain-rate, inherent in the Navier-Stokes theory, might not always be appropriate for the regions of large strain rate that occur as a flow makes the transition to turbulence. He considered a weakly non-Newtonian situation, in which a second term needs to be added to the Navier-Stokes system, to model the effects of (memoryless) visco-elasticity. He demonstrated that, in such models, the flow cannot remain stable to small disturbances for all Reynolds numbers. Instead, there is a transition value at which a very large number of the eigenmodes all become unstable; furthermore, their frequencies are not rational multiples of one another so that, even in linearized (small disturbance) theory, the resulting flow behavior would contain a quasi-periodic structure of extremely high dimension. Non-linear effects would then cause this structure to bifurcate to a strange attractor of high dimension [through the mechanism of a Ruelle-Takens-Newhouse bifurcation; see Ref. 5 (p. 339)]. Forbes ${ }^{1,2}$ and Forbes and Brideson ${ }^{3}$ suggested that this exotic, high-dimensional chaotic behavior may correspond to the onset of true turbulence.

As a possible viscometric flow for measuring the two viscosity parameters, the Reynolds number $R_{e}$ and the nonlinear viscosity coefficient $F$, we are currently investigating slow flow between rotating disks with a view to its laboratory implementation. This is now a classical flow with a famous similarity-type solution first suggested by von Kármán. ${ }^{6} \mathrm{He}$ showed that, when the Navier-Stokes equations of viscous

a)Larry.Forbes@utas.edu.au fluid flow are written in cylindrical polar coordinates, a separable solution is possible, in which the steady-state velocity components and the pressure can each be expressed as simple powers of the radial coordinate $r$ multiplied by functions of the vertical coordinate $z$. These functions of $z$ satisfy a system of non-linear ordinary differential equations. An enormous literature now exists on this von Kármán rotation, much of which is discussed in the reviews by Zandbergen ${ }^{7}$ and Lingwood. ${ }^{8}$ From the viscometric viewpoint, the flow of most interest is likely to be the situation in which there are two parallel disks with the fluid between them; the top disk at $z=H$ rotates with constant angular speed $\Omega \mathrm{rad} / \mathrm{s}$, but the bottom disk at $z=0$ is stationary since this would allow pressure transducers to be attached to that plate. This situation has been of great interest in the literature, particularly due to the difference of opinion between Batchelor and Stewartson concerning the behavior of the fluid. Batchelor ${ }^{9}$ suggested that the flow configuration would consist of constant angular speed motion between the plates and a boundary layer near each plate. However Stewartson ${ }^{10}$ predicted no rotation in the core, but boundary layers near each plate. It is now known that multiple solutions to the steady von Kármán problem are possible; these are illustrated by Holodniok and Hlaváček, ${ }^{11}$ although their numerical results are by no means independent of the number of Fourier modes assumed in their numerical solution.

The interest here is in a Reiner-Rivlin model of viscous fluid flow, rather than the more common Navier-Stokes equations. This is because it is the simplest memoryless model for viscous behavior, which does not assume a linear relationship between the stress tensor in the fluid and the strain-rate tensor. Thus it may have relevance to the process of flow transitioning from laminar to turbulent, and further details are given in the work of Forbes. ${ }^{1}$ It turns out that a von Kármán type similarity solution is also possible in this case, too, as was apparently first recognized by Bhatnagar. ${ }^{12}$ More recently, it has been shown that von Kármán type similarity solutions are also possible in more general non-Newtonian fluids (see Refs. 13 and 14). These articles do not discuss the possibility of multiple solutions, although multiplicity is mentioned 
for general non-Newtonian fluids in the review article by Rajagopal. ${ }^{15}$

The governing equations are reviewed in Sec. II, where an appropriate non-dimensionalization is discussed and the spectral solution method is outlined. Results are discussed in Sec. III. The possible use of this flow for accurate viscometric work in the laboratory is discussed in Sec. IV, particularly at low Reynolds numbers and in the light of the existence of multiple solutions.

\section{THE GOVERNING EQUATIONS}

We consider a flat stationary disk on the $x-y$ plane of a Cartesian coordinate system in which the $z$-axis points vertically. There is another flat disk at height $z=H$, and it rotates with angular speed $\Omega \mathrm{rad} / \mathrm{s}$. Between the two disks is a Reiner-Rivlin viscous fluid, and it is subject to the acceleration of gravity $g$ downwards, in the direction of the negative $z$-axis. The fluid has constant density $\rho$ since it is assumed incompressible, and its velocity vector is $\mathbf{q}$.

Dimensionless variables are defined forthwith, using the disk separation distance $H$ as the length scale and $\sqrt{H / g}$ as the unit of time. Then speeds are referenced to $\sqrt{g H}$, and the pressure $p$ in the fluid is made dimensionless with respect to the quantity $\rho g H$. The equations of motion are expressed in cylindrical polar coordinates $(r, \theta, z)$ according to the usual relations $x=r \cos \theta, y=r \sin \theta$, and new unit vectors $\left(\mathbf{e}_{\mathbf{r}}, \mathbf{e}_{\boldsymbol{\theta}}, \mathbf{e}_{\mathbf{z}}\right)$ in the three coordinate directions are defined. The fluid velocity vector can now be represented as $\mathbf{q}=u \mathbf{e}_{\mathbf{r}}+v \mathbf{e}_{\boldsymbol{\theta}}+w \mathbf{e}_{\mathbf{z}}$. Nondimensionalization shows that the problem is described by three dimensionless parameters

$$
\frac{1}{R_{e}}=\frac{\mu}{\rho H \sqrt{g H}}, \quad \frac{1}{F}=\frac{\tau}{\rho H^{2}}, \quad \omega=\Omega \sqrt{\frac{H}{g}} .
$$

Here, $R_{e}$ is a Reynolds number, $1 / F$ is the coefficient of nonNewtonian viscosity, and $\omega$ is the dimensionless rotation speed of the top plate (now at $z=1$ ). The dimensional constants $\mu$ and $\tau$ are, respectively, the Newtonian and non-Newtonian viscosity coefficients, here assumed constant. It is possible to write parameter $F$ in terms of the more common Weissenberg and Reynolds numbers, ${ }^{1}$ but this is avoided here since $F$ is a pure measure of non-Newtonian behavior in the fluid.

Since the fluid is incompressible, the continuity equation is

$$
\nabla \cdot \mathbf{q}=0
$$

The fundamental law expressing conservation of linear momentum within a continuous medium is Cauchy's momentum equation

$$
\frac{\partial \mathbf{q}}{\partial t}+(\mathbf{q} \cdot \nabla) \mathbf{q}=\mathbf{f}+\operatorname{div} \mathbf{T}
$$

in these dimensionless variables, in which $\mathbf{T}$ is the stress tensor in the continuum. In Navier-Stokes theory, stress is assumed to vary linearly with the strain-rate tensor $\mathbf{D}$ according to the relation

$$
\mathbf{T}=-p \mathbf{I}+\frac{2}{R_{e}} \mathbf{D}
$$

in which $p$ is the (dimensionless) pressure in the fluid and $R_{e}$ is the Reynolds number as defined in (1). The $3 \times 3$ tensor $\mathbf{I}$ is the identity matrix. This linear assumption is basic to NavierStokes theory, but Stokes himself allowed that a non-linear stress-strain-rate relation might also be considered, leading to a constitutive law of the form

$$
\mathbf{T}=-p \mathbf{I}+\frac{2}{R_{e}} \mathbf{D}+\frac{2}{F} \mathbf{D}^{2},
$$

with the second viscosity coefficient $1 / F$ given in (1). This relation (5) is discussed in detail by Aris. ${ }^{16}$ It is, in fact, the general form of a non-linear stress-strain-rate law of the type $\mathbf{T}=f(\mathbf{D})$ for any non-linear analytic function $f(Z)$ that can be represented by a Taylor-MacLaurin series since, by the CayleyHamilton theorem [see Ref. 17 (p. 183)], every higher power $\mathbf{D}^{k}, k=3,4,5, \ldots$, of the $3 \times 3$ tensor $\mathbf{D}$ can be written as a linear combination of the three matrices $\mathbf{I}, \mathbf{D}$, and $\mathbf{D}^{2}$ in Eq. (5). The combination of (5) and (3) then leads to the Reiner-Rivlin equation

$$
\frac{\partial \mathbf{q}}{\partial t}+(\mathbf{q} \cdot \nabla) \mathbf{q}+\nabla p=-\mathbf{e}_{\mathbf{z}}+\frac{1}{R_{e}} \nabla^{2} \mathbf{q}+\frac{2}{F} \operatorname{div}\left(\mathbf{D}^{2}\right),
$$

in which $\mathbf{D}$ is the rate-of-strain tensor,

$$
\mathbf{D}=\frac{1}{2}\left[\nabla \mathbf{q}+(\nabla \mathbf{q})^{T}\right]
$$

In these dimensionless variables, the body force per mass appropriate for the gravitational acceleration becomes simply $\mathbf{f}=-\mathbf{e}_{\mathbf{z}}$ as shown in (6) above.

On the stationary bottom plate, the no-slip boundary condition gives

$$
u=0, \quad v=0, \quad w=0, \quad \text { on } z=0 .
$$

The no-slip boundary condition on the steadily rotating top disk gives

$$
u=0, \quad v=r \omega, \quad w=0, \quad \text { on } z=1 .
$$

The celebrated von Kármán similarity solution, ${ }^{6}$ extended by Bhatnagar ${ }^{12}$ to allow for non-Newtonian effects in the Reiner-Rivlin equation (6), takes the form

$$
\begin{aligned}
u(r, z, t) & =r U(z, t), \\
v(r, z, t) & =r V(z, t), \\
w(r, z, t) & =W(z, t), \\
p(r, z, t) & =P(z, t)+r^{2} Q(z, t) .
\end{aligned}
$$

Steady flow is now assumed so that the time variable $t$ no longer appears. Accordingly, when (10) are substituted into the continuity equation (2), they give the ordinary differential equation

$$
2 U+W^{\prime}=0
$$

In cylindrical polar coordinates $(r, \theta, z)$, the three components of the Reiner-Rivlin equation (6) yield four ordinary differential equations since the $z$-component equation involves two linearly independent functions $r^{0}$ and $r^{2}$ of the radial coordinate, which must be treated separately. These can then be integrated with respect to $z$ at once, to give 


$$
\begin{aligned}
& P(z)=P_{0}-z+\left(1 / R_{e}\right) W^{\prime}-(1 / 2) W^{2}+(7 / F) U^{2}, \\
& Q(z)=k_{Q S}+\frac{1}{2 F}\left[\left(U^{\prime}\right)^{2}+\left(V^{\prime}\right)^{2}\right],
\end{aligned}
$$

in which $P_{0}$ and $k_{Q S}$ are both constants of integration. The first constant $P_{0}$ plays no role of importance and so can be ignored, but the second $k_{Q S}$ is an eigenvalue and must be determined as part of the steady solution. The radial and azimuthal components of (6) become

$$
\begin{aligned}
& U^{2}-V^{2}+W U^{\prime}+2 k_{Q S} \\
& \quad=\left(1 / R_{e}\right) U^{\prime \prime}-\frac{1}{2 F}\left[\left(U^{\prime}\right)^{2}+3\left(V^{\prime}\right)^{2}+2 U U^{\prime \prime}\right], \\
& 2 U V+W V^{\prime} \\
& \quad=\left(1 / R_{e}\right) V^{\prime \prime}+(1 / F)\left[U^{\prime} V^{\prime}-U V^{\prime \prime}\right] .
\end{aligned}
$$

The quantity $Q(z)$ has already been eliminated in the first of these equations, using (12).

The two boundary conditions (8) and (9) yield the six requirements

$$
\begin{aligned}
& U(0)=0, \quad V(0)=0, \quad W(0)=0, \\
& U(1)=0, \quad V(1)=\omega, \quad W(1)=0,
\end{aligned}
$$

which must be obeyed by the non-linear steady solution. To account for these conditions (14), we choose the spectral forms

$$
\begin{aligned}
& U(z)=\sum_{n=1}^{N} A_{n} \sin (n \pi z), \\
& V(z)=\omega z+\sum_{n=1}^{N} B_{n} \sin (n \pi z) .
\end{aligned}
$$

The sums in these expressions should consist of infinitely many terms, but in numerical implementation, they must be truncated at some maximum number $N$ of Fourier modes, as indicated in (15). The Fourier coefficients $A_{n}$ and $B_{n}$ are to be determined. The continuity equation (11) then requires

$$
W(z)=-\sum_{n=1}^{N} A_{n} \frac{2}{n \pi}[1-\cos (n \pi z)],
$$

after the condition $W(0)=0 \mathrm{in}(14)$ has been taken into account. When the condition $W(1)=0$ on the top boundary is imposed, it follows that

$$
A_{1}=-\frac{1}{2} \sum_{n=2}^{N} A_{n} \frac{1}{n}[1-\cos (n \pi)] .
$$

Thus only the $N-1$ coefficients $A_{2}, \ldots, A_{N}$ are independent.

These series expressions (15)-(17) are substituted into the two equations in the system (13) and Fourier analyzed using the orthogonality of the trigonometric functions. The radial momentum equation yields

$$
\begin{aligned}
& \frac{1}{2 R_{e}} A_{\ell}(\ell \pi)^{2}+\frac{2 k_{Q S}}{\ell \pi}[1-\cos (\ell \pi)] \\
& +\int_{0}^{1}\left[U^{2}-V^{2}+W U^{\prime}\right] \sin (\ell \pi z) d z \\
& +\frac{1}{2 F} \int_{0}^{1}\left[\left(U^{\prime}\right)^{2}+3\left(V^{\prime}\right)^{2}+2 U U^{\prime \prime}\right] \sin (\ell \pi z) d z=0, \\
& \ell=1, \ldots, N,
\end{aligned}
$$

and the azimuthal component gives

$$
\begin{gathered}
\frac{1}{2 R_{e}} B_{\ell}(\ell \pi)^{2}+\int_{0}^{1}\left[2 U V+W V^{\prime}\right] \sin (\ell \pi z) d z \\
+\frac{1}{F} \int_{0}^{1}\left[U V^{\prime \prime}-U^{\prime} V^{\prime}\right] \sin (\ell \pi z) d z=0 \\
\ell=1, \ldots, N
\end{gathered}
$$

These two sets of Eqs. (18) and (19) constitute a system of $2 N$ non-linear algebraic equations for the $2 \mathrm{~N}$-vector of unknowns,

$$
\mathbf{X}=\left[k_{Q S}, A_{2}, \ldots, A_{N}, B_{1}, B_{2}, \ldots, B_{N}\right]^{T} .
$$

This system is solved using Newton's method.

\section{PRESENTATION OF RESULTS}

A typical result from the spectral solution technique of Sec. II is illustrated in Fig. 1. Here, the Reynolds number is $R_{e}=10^{3}$ and the non-linear viscosity coefficient is $F=10^{4}$. These are highly accurate and well-converged solutions generated with $N=81$ Fourier modes. In addition, 801 grid points in $z$ were used to carry out the numerical integration, using the Gaussian quadrature package lgwt written by von Winckel. ${ }^{18}$ The eigenvalue $k_{Q S}$ is plotted against rotation speed $\omega$, and it is evident that up to three solution branches exist for appropriate rotation speeds $\omega$. The corresponding calculation has also been done for the pure Navier-Stokes flow, with $F=\infty$, and the graph in that case is almost identical to Fig. 1, and so it is not shown here.

In Fig. 2, we show the velocity function $U(z)$ associated with the radial outflow component $u(r, z)$ of the velocity vector, as defined in (10). These three diagrams are for the same case $R_{e}=10^{3}, F=10^{4}$ as in Fig. 1. Here, the rotation rate has been chosen to be $\omega=0.5$, which corresponds to a vertical line at the very right edge of Fig. 1. The result in Fig. 2(a) is for the bottom branch in Fig. 1 and shows a sharp flow of fluid

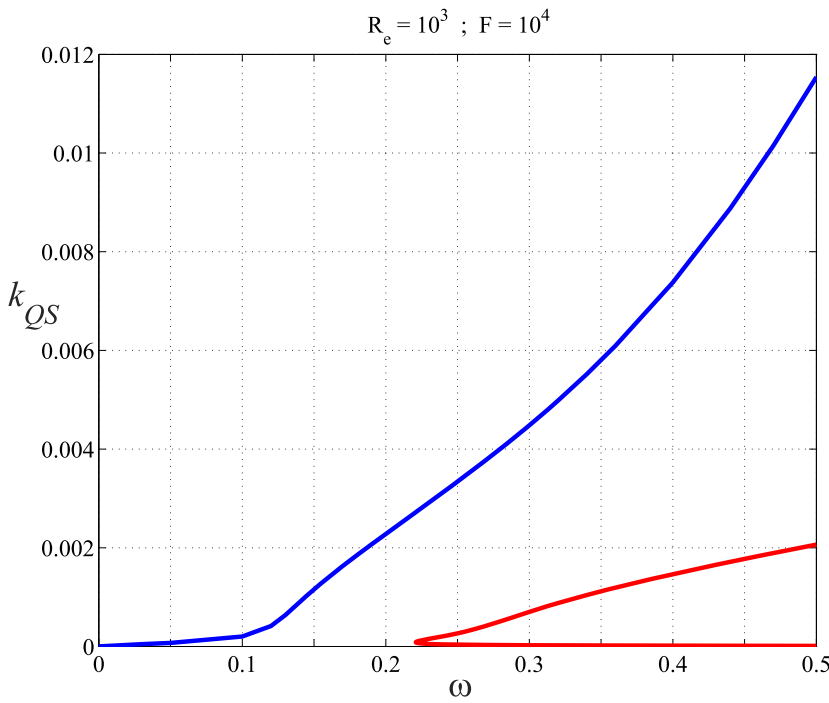

FIG. 1. Dependence of the non-linear eigenvalue $k_{Q S}$ on rotation rate $\omega$, for Reynolds number $R_{e}=1000$ and $F=10000$. 


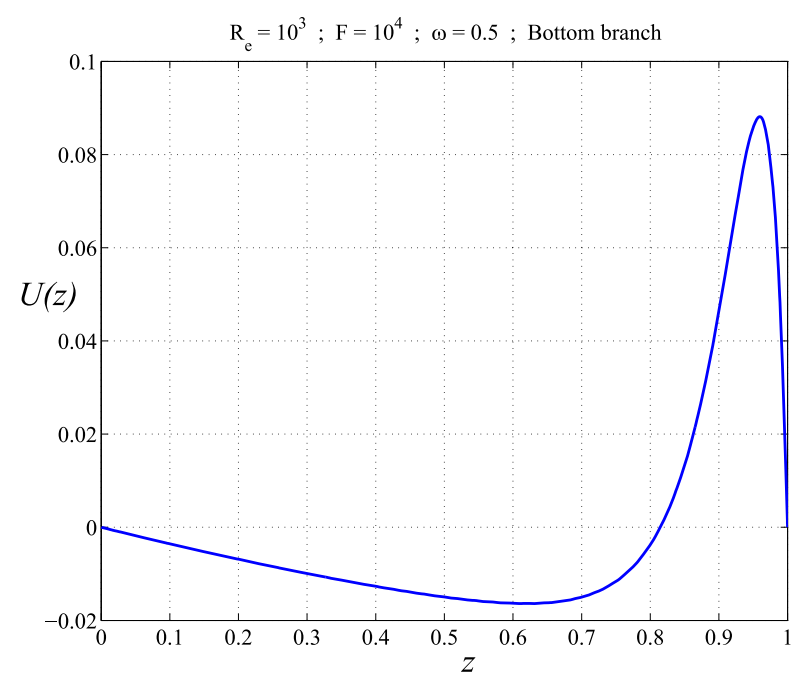

(a)

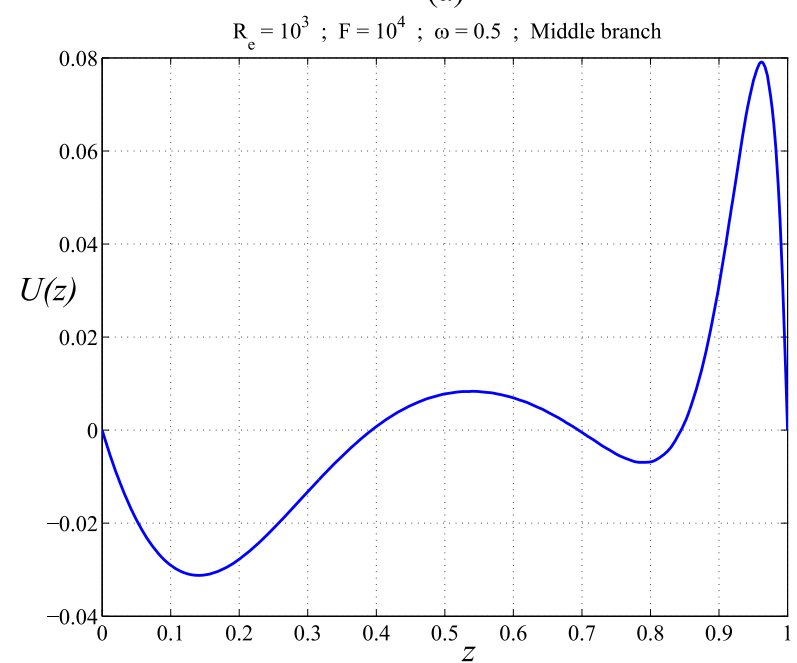

(b)

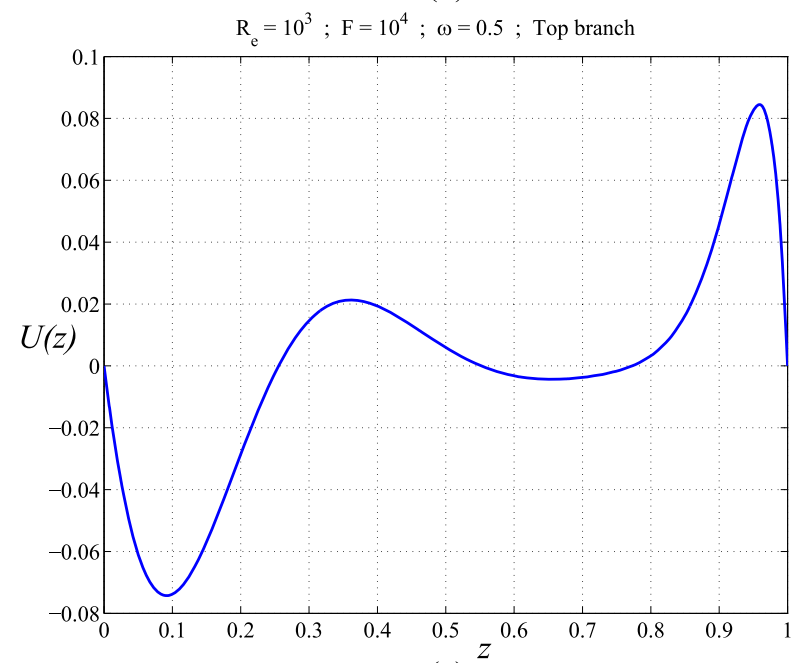

(c)

FIG. 2. The radial velocity function $U(z)$ for the three solution branches illustrated in Fig. 1, with (a) bottom branch, (b) middle branch, and (c) top branch. The rotation rate is $\omega=0.5$, and the Reynolds number $R_{e}=1000$ and $F=10000$.

radially outwards in a narrow boundary layer out along the top plate near $z=1$. Below this layer, over the approximate interval $0<z<0.85$, the function $U(z)$ is small and negative, representing a slow inward movement of the fluid back inwards toward the centre, as is required by the conservation of mass. For this bottom solution branch, therefore, the most dramatic outflows occur in the upper boundary layer near the top plate. For the top branch in Fig. 1, illustrated in Fig. 2(c) at rotation speed $\omega=0.5$, there is still the strong outflow in a narrow boundary layer out along the top plate $z=1$, but for this branch, there is also a sharp inflow towards the centre, in a boundary layer near the bottom plate at $z=0$. In a region near the middle section $z=0.5$ between the two plates, there is almost no radial flow, with $U \approx 0$. The middle branch illustrated in part (b) also has radial outflow occurring in a narrow boundary layer near the moving top plate, but also has a broad region of inwardly directed return flow over the approximate interval $0<z<0.4$ of the fluid layer.

The continuity equation (2) can be satisfied identically using a vector potential $\mathbf{A}(r, z)$, and without loss of generality, this may be assumed to have only two components $\Psi$ and $\Lambda$ so that

$$
\mathbf{A}=\Psi \mathbf{e}_{\boldsymbol{\theta}}+\Lambda \mathbf{e}_{\mathbf{z}}
$$

The velocity vector can be written as $\mathbf{q}=\nabla \times \mathbf{A}$, and since this axisymmetric flow has no $\theta$-dependence, the three velocity components in the radial, azimuthal, and vertical directions become

$$
u=-\frac{\partial \Psi}{\partial z}, \quad v=-\frac{\partial \Lambda}{\partial r}, \quad w=\frac{1}{r} \frac{\partial}{\partial r}(r \Psi)
$$

in these cylindrical polar coordinates. When these formulae (22) are combined with the von Kármán similarity forms (10), they lead to the expressions

$$
\Psi(r, z)=\frac{1}{2} r W(z), \quad \Lambda(r, z)=-\frac{1}{2} r^{2} V(z)
$$

for the two streamfunctions $\Psi(r, z)$ and $\Lambda(r, z)$. Forbes and Brideson $^{3}$ also made use of a bi-streamfunction formulation similar to (23) in their analysis of turbulence in a rotating circular pipe. It follows that, since the radial and vertical velocity components $u$ and $w$ in Eq. (22) are independent of the second streamfunction $\Lambda$, it is possible to view axisymmetric streamsurfaces as cross sections in the $(r, z)$ plane by considering the first streamfunction $\Psi$ only.

The velocity vector $\mathbf{q}$ is required to be tangent everywhere to such a streamsurface, and this consideration gives rise to the defining equation

$$
\frac{d r}{d z}=\frac{u}{w}
$$

for the streamsurface. It follows then from the similarity forms (10) and the definitions (23) that

$$
r \Psi(r, z)=\frac{1}{2} r^{2} W(z)=\text { constant on a streamsurface. }
$$

Figure 3 shows cross sections of some streamsurfaces for the same Reynolds number $R_{e}=1000$ and non-linear viscosity parameter $F=10000$ as in Fig. 1. The three diagrams presented here were obtained by drawing contours of the function $r \Psi$ in (25). In addition, the velocity vector $u \mathbf{e}_{\mathbf{r}}+w \mathbf{e}_{\mathbf{z}}$ in the vertical plane is indicated by the field of arrows in each diagram. The lengths of these arrows are an indication of the relative magnitudes of the velocities at each point. The rotation rate is $\omega=0.5$, as was considered in Fig. 2. The flow pattern for 


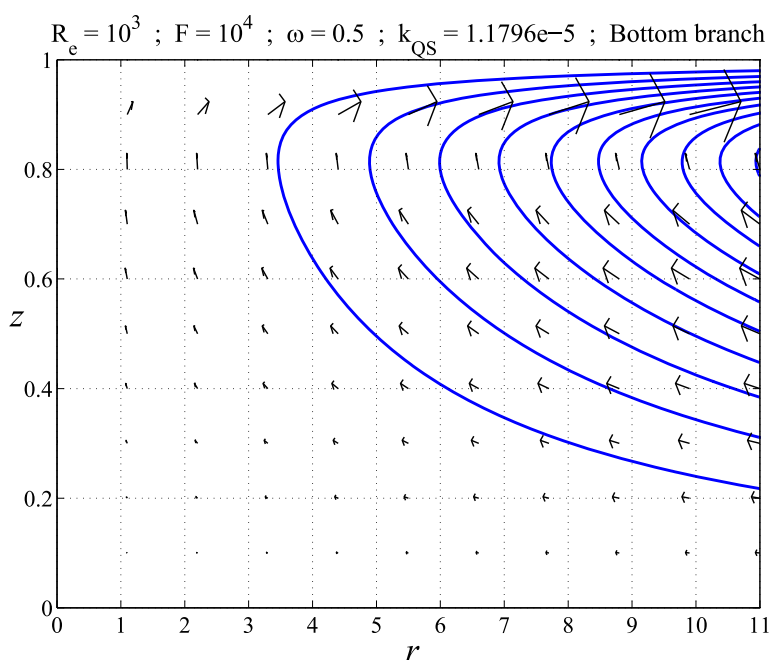

(a)

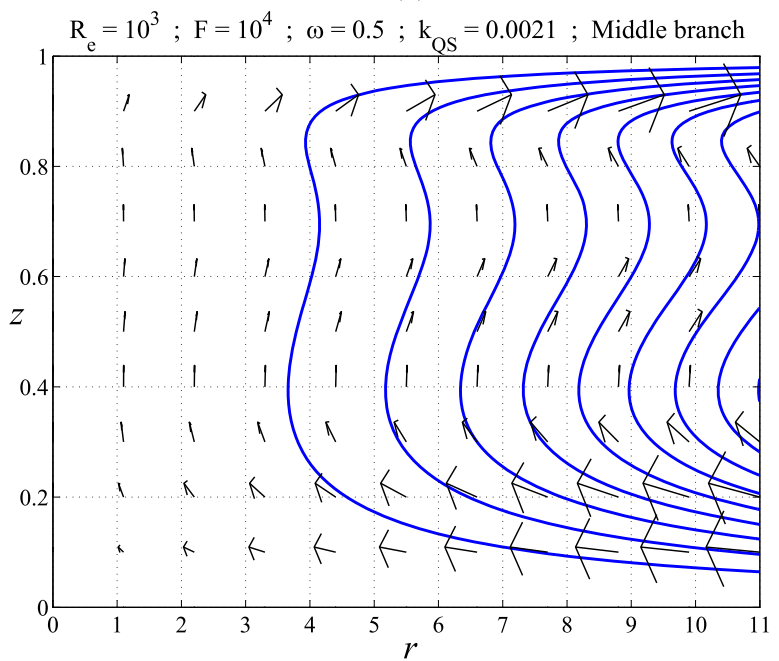

(b)

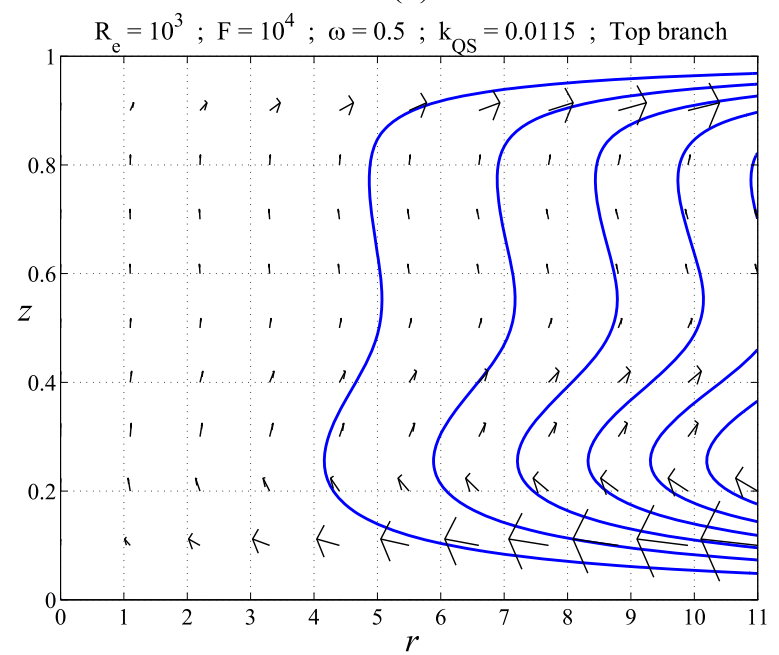

(c)

FIG. 3. Streamsurfaces in $(r, z)$ space for (a) bottom branch, (b) middle branch, and (c) top branch solutions, for the same parameter values $R_{e}=1000$ and $F=10000$ as in Fig. 1 and with rotation rate $\omega=0.5$.

the lowest solution branch is shown in Fig. 3(a). It indicates a strong outflow near the top wall at $z=1$ and a much weaker return inflow over the rest of the fluid region. This is consistent with the diagram of the radial velocity function $U(z)$ shown for this same solution branch in Fig. 2(a). The middle and top branch streamline patterns are shown in Figs. 3(b) and 3(c), respectively, and the solution for the top branch in part (c), in particular, indicates an outflow boundary layer near the top plate, with a corresponding inflow in a boundary near the stationary bottom plate at $z=0$. This again confirms the result shown in Fig. 2(c).

The azimuthal velocity function $V(z)$ is shown in Fig. 4, for the three solution branches illustrated in Fig. 1, at rotation speed $\omega=0.5$ (at the right-most edge of Fig. 1). This diagram confirms strongly that the bottom branch at the right side of Fig. 1 is indeed a Stewartson-type solution, since there is no rotation at all in the core, and a boundary layer to the right of the diagram, in which the speed rises sharply to its value $V=0.5$ on the top plate at $z=1$. By contrast, the top-branch solution illustrates clearly an azimuthal flow consistent with the predictions of Batchelor; ${ }^{9}$ there is no flow at the stationary bottom disk, but the rotation speed rises rapidly to a nearly constant value of about $V \approx 0.16$ in the region between the disks. Finally, the rotational speed rises sharply to its eventual value $V=0.5$, in a narrow boundary layer at the top plate.

The middle-branch solution is interesting, for it consists of reverse rotation over most of the fluid domain between the plates, returning to zero only at the height $z \approx 0.84$, after which it rises rapidly to the speed $V=0.5$ at the rotating disk, in a narrow boundary layer. A solution of this type, with a large region of slow reverse rotation, is undoubtedly unstable. This has not been pursued here, however, since the far more interesting stability question necessarily involves a consideration of non-axisymmetric flow geometry, which is outside the scope of this present paper.

The pressure in the fluid is illustrated in Fig. 5, for the same parameter values as shown in Fig. 3. Only the bottom and top solution branches are shown, since the middle solution branch is anticipated to be unstable, and so would not be seen in the laboratory. For the bottom-branch solution in Fig. 5(a), the pressure at the lower plate $z=0$ essentially does not change with radius $r$, similar to the pure Navier-Stokes solution. This

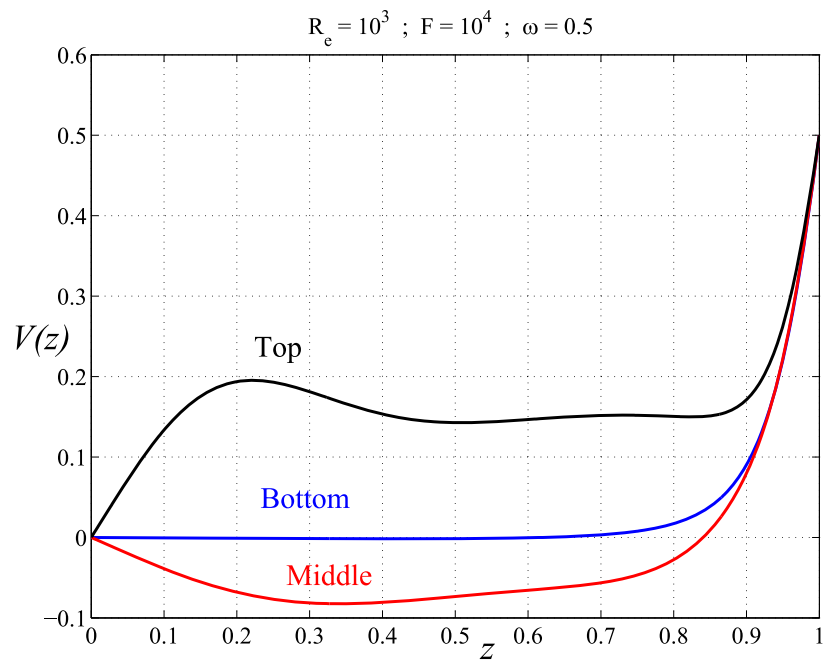

FIG. 4. The azimuthal velocity function $V(z)$ for the three solution branches illustrated in Fig. 1, for rotation rate $\omega=0.5$ with Reynolds number $R_{e}=1000$ and $F=10000$. 


$$
\mathrm{R}_{\mathrm{e}}=10^{3} ; \mathrm{F}=10^{4} ; \omega=0.5 ; \text { Bottom Branch }
$$

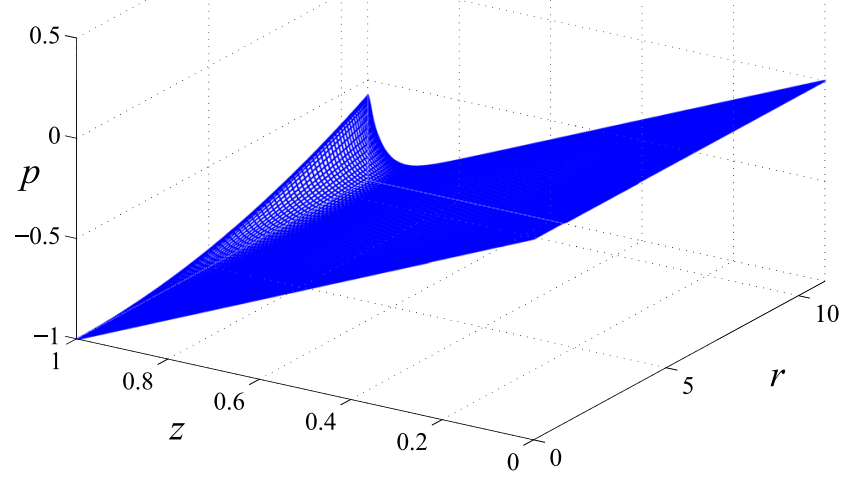

(a)

$$
\mathrm{R}_{\mathrm{e}}=10^{3} ; \mathrm{F}=10^{4} ; \omega=0.5 ; \text { Top Branch }
$$

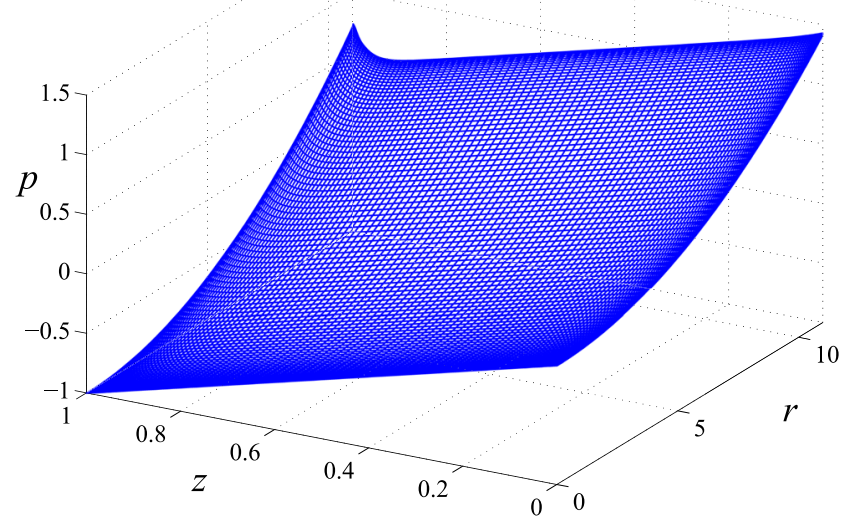

(b)

FIG. 5. Pressure $p$ as a function of $r$ and $z$, for (a) the bottom branch and (b) the top branch solutions, for the case illustrated in Fig. 3, in which $R_{e}=1000$ and $F=10000$ with rotation rate $\omega=0.5$.

is because the function $Q(z)$ in Eq. (10) is extremely small $\left(Q \approx 1.2 \times 10^{-5}\right)$ and so makes no appreciable contribution to the overall pressure. From the viscometric point of view, this solution branch is therefore of little interest since the experimental determination of the two viscosity parameters $R_{e}$ and $F$ in the way discussed in the Introduction would rely on measurable differences in the pressure $p$ with radius $r$ on the bottom plate. For the top solution branch shown in Fig. 5(b), however, the variation of pressure $p$ with radius $r$ across the bottom plate is significant (since $Q \approx 0.0115$ ), making viscometric determination of the nonlinear viscosity parameter $F$ a possibility. This is discussed further in Sec. IV.

The pressure on the non-rotating bottom plate is sensitive to the Reynolds number, which in Figs. 1-5 has been set to the value $R_{e}=1000$. It is of interest now to consider a lower Reynolds number, and accordingly, we present in Fig. 6 a similar bifurcation diagram to Fig. 1, but now at the much lower Reynolds number $R_{e}=5$. The viscoelastic parameter remains at its previous value $F=10^{4}$. Solutions are now shown for larger rotation speeds $\omega$, and the eigenvalue $k_{Q S}$ also takes

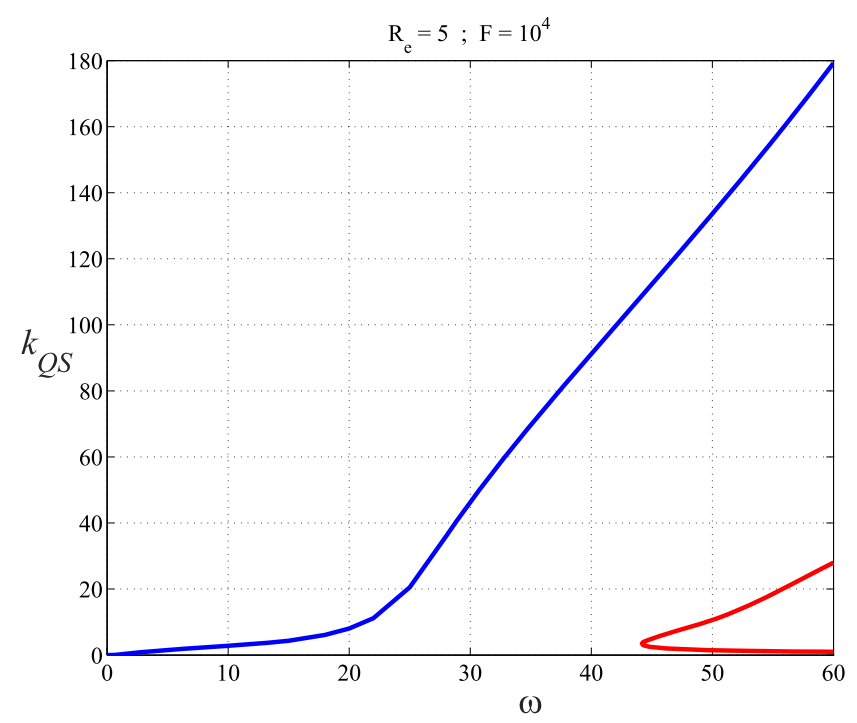

FIG. 6. Dependence of the non-linear eigenvalue $k_{Q S}$ on rotation rate $\omega$, for Reynolds number $R_{e}=5$ and $F=10000$.

correspondingly larger values. Again there are three solution branches that have been found, and these are highly accurate numerical results.

Streamline patterns are illustrated in Fig. 7 for this case $R_{e}=5, F=10^{4}$, for the three different solution branches that have been obtained at the rotation rate $\omega=60$ at the right-most point of Fig. 6. Similar to Fig. 3, the bottom-branch solution shown in Fig. 7(a) is of Stewartson type, with only slight motion near the bottom plate but a strong outflow in a boundary layer confined to a region near the rotating top plate. The top-branch solution in (c), however, is of the Batchelor type, with inflow along the bottom plate and outflow near the top and an approximately constant flow region in the core. This is again confirmed by an inspection of the azimuthal velocity function $V(z)$, which is qualitatively similar to the results shown in Fig. 4 for each of the solution branches. The middlebranch solution again has a large core region of slow reverse rotation, suggesting again that it would be unstable even within the confines of this axisymmetric flow geometry.

From the viscometric point of view, the function $Q(z)$ appearing in (10) as part of the overall expression for pressure is of particular interest. For pure Navier-Stokes flow, $Q(z)=0$ so that non-zero values of this function provide a direct measure of the non-Newtonian effects within the fluid. Figure 8 shows the function $Q(z)$ for each of the three solution branches at the right-most section of Fig. 6, for angular speed $\omega=60$. In each case, the function $Q(z)$ is nearly constant for much of the fluid domain between the plates, although it rises slightly in the region of the boundary layer near the top plate at $z=1$. The bottom branch gives a function that is so small that it would be almost indistinguishable from pure Newtonian fluid; while the values of $Q(z)$ associated with the middle branch are significantly larger, this branch would be unstable and therefore also of little value in viscometry. The top branch, however, possesses a pressure deviation function $Q(z)$ that is large enough to be of significance. As a result, this Batchelor-type solution would be preferred as a way of measuring the viscoelastic parameter $F$ using wall-mounted pressure 


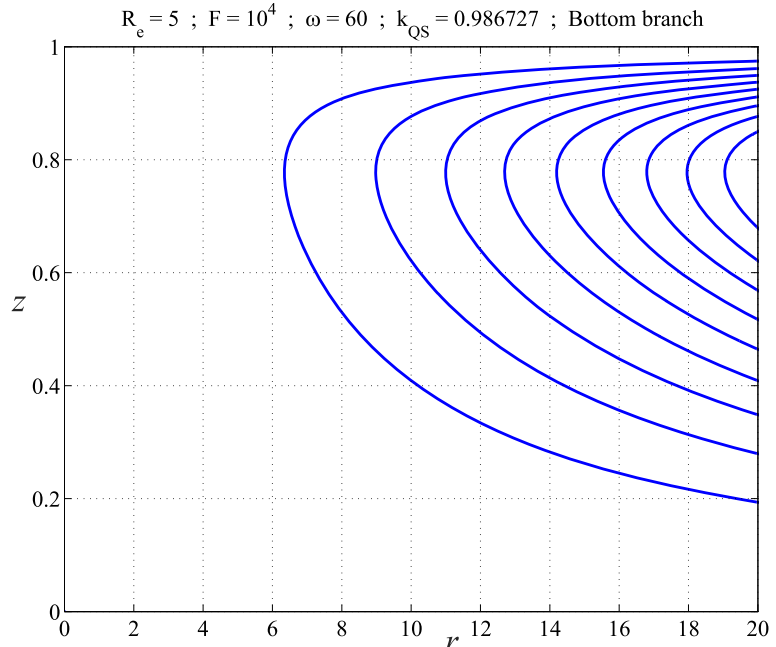

(a)

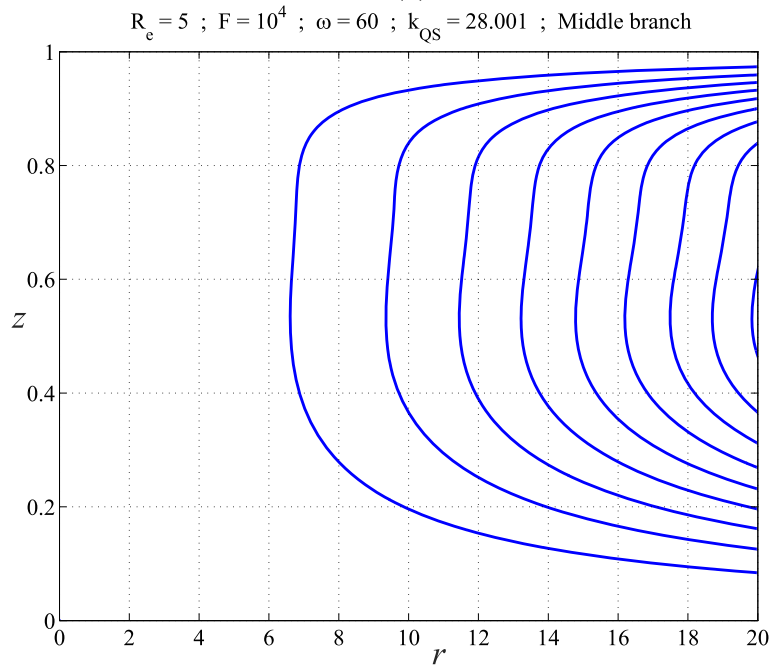

(b)

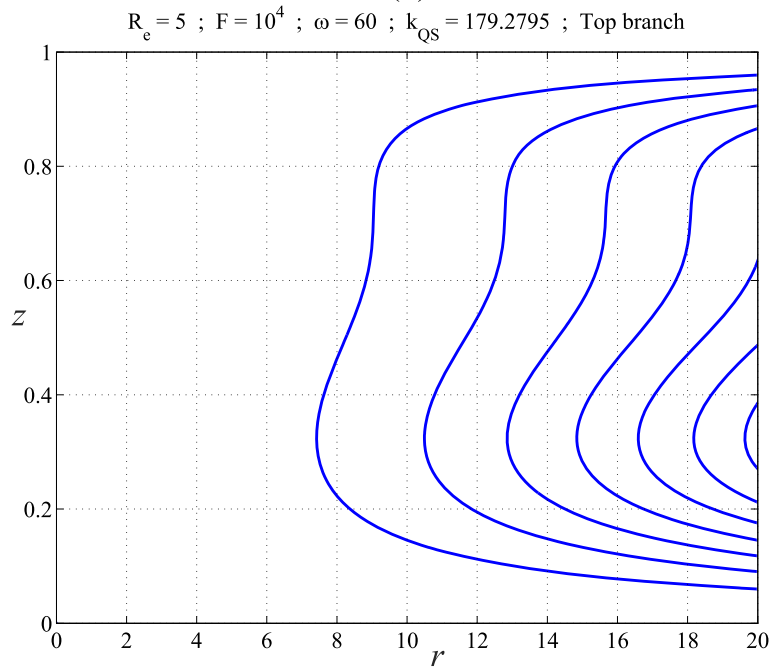

(c)

FIG. 7. Streamsurfaces in $(r, z)$ space for (a) bottom branch, (b) middle branch, and (c) top branch solutions, for the same parameter values $R_{e}=5$ and $F=10000$ as in Fig. 6 and with rotation rate $\omega=60$.

transducers and hence obtaining the value of the dimensional quantity $\tau$ in (1).

Figure 9 presents the dependence of the eigenvalue $k_{Q S}$ on the coefficient $1 / F$ of the non-linear viscosity term in the

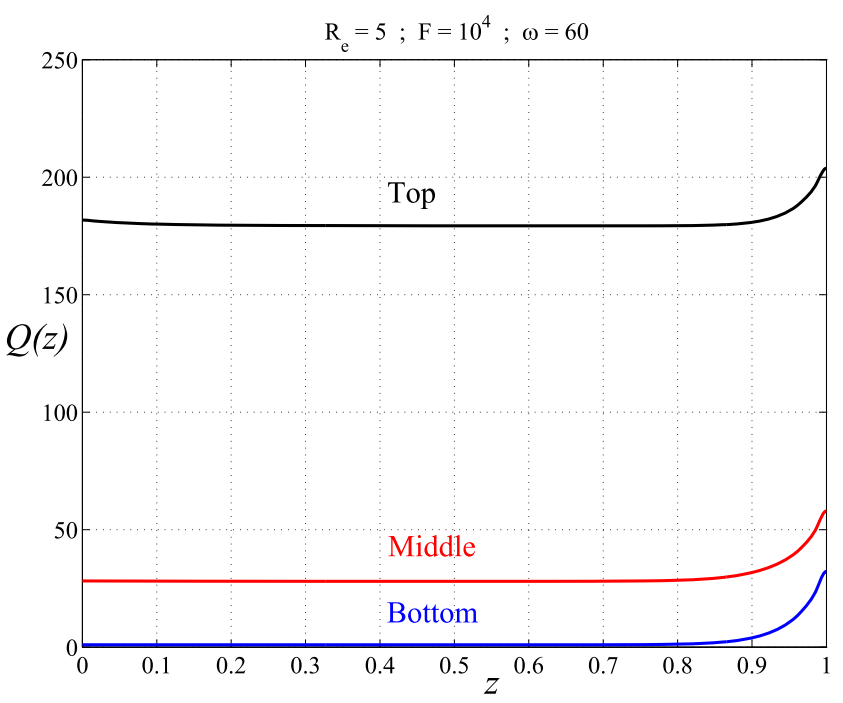

FIG. 8. Behavior of the non-Newtonian pressure component $Q(z)$ with height $z$, for Reynolds number $R_{e}=5$ and $F=10000$ and at rotation rate $\omega=60$.

Reiner-Rivlin equation (6). The vertical axis at $1 / F=0$ is the pure Navier-Stokes case, and the three lines that intersect it correspond to the three solution branches for $\omega=0.5$ at values of $k_{Q S}$ equal to those shown on the right-most edge of Fig. 1. Thus the portion of Fig. 9 to the right of the $k_{Q S}$-axis represents shear-thickening Reiner-Rivlin fluids with $F>0$, whereas the region to the left of the axis corresponds to shear-thinning fluids with $F<0$. As these results were being generated by the solution algorithm described in Sec. II, it was anticipated that perhaps these two branches that exist in the shear-thinning region $1 / F<0$ might eventually coalesce, perhaps joining in a fold bifurcation, but Fig. 9 shows conclusively that this is not what happens. Instead, the two solution branches meet and exchange positions at about $1 / F=-2.6$, as may be seen in Fig. 9. In spite of the appearance of these two solution branches

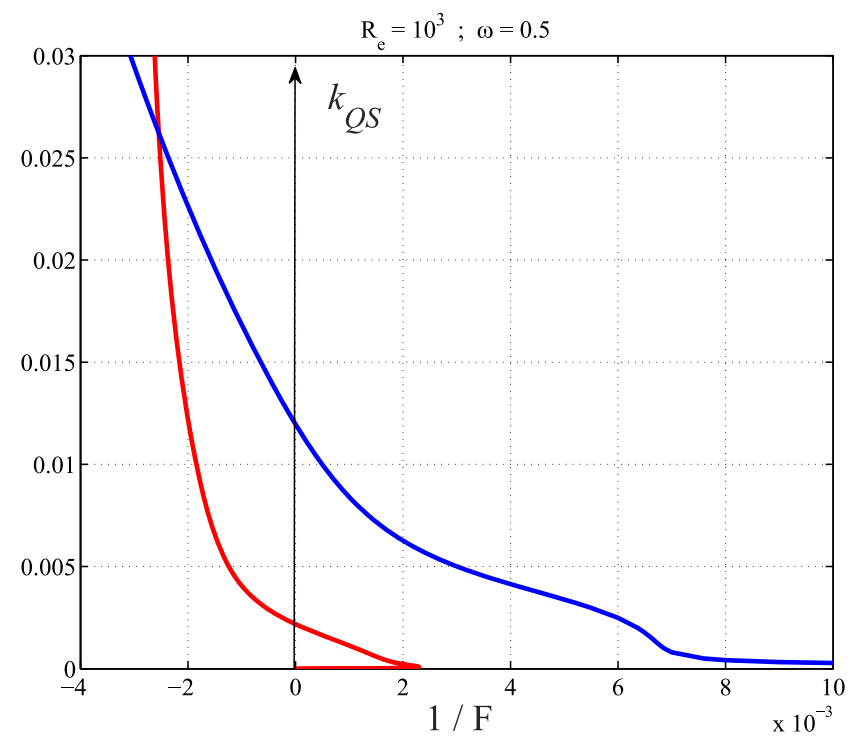

FIG. 9. Bifurcation diagram for the Stewartson (red line, bottom branch) and Batchelor (blue line, top branch) type solutions, for Reynolds number $R_{e}=1000$ and at rotation rate $\omega=0.5$. The dependence of the eigenvalue $k_{Q S}$ on the viscoelastic coefficient of the non-Newtonian viscosity term is shown. 
in Fig. 9, this point does not correspond to a transcritical bifurcation since it occurs in the much higher-dimensional space that includes the Fourier coefficients in the representation (15) through the vector (20).

\section{CONCLUSIONS}

This paper has considered the famous von Kármán similarity form of the equations for viscous flow between two flat plates, one of which rotates and the other is stationary. This is a topic which has received an enormous amount of attention, primarily for Navier-Stokes flow, but here the interest is in a weakly non-Newtonian fluid described by Reiner-Rivlin theory. As indicated in the Introduction, a partial reason for this is to test empirically the theory of the transition to turbulence propounded by Forbes ${ }^{1}$ and colleagues. Highly accurate solutions have been generated using a spectral method that has the somewhat novel feature that one of the series coefficients must be determined in terms of the others and that an integration constant $k_{Q S}$ then serves as an eigenvalue for the nonlinear problem. As indicated in the Introduction, the Reiner-Rivlin fluid can be regarded as the simplest model of a nonlinear stress-strain-rate law in a visco-elastic fluid without memory, and Forbes ${ }^{1}$ suggested that all such fluids may undergo a transition to turbulent flow behavior that is at least qualitatively equivalent to the highly complex elasticinertial instability that occurs in the Reiner-Rivlin equation. Purely Navier-Stokes fluids would generate constant pressure across the non-rotating bottom plate, and so any variation in pressure would be the evidence of fluid material nonlinearity.

As anticipated from studies with Newtonian fluids (Ref. 11) and some non-Newtonian fluids (Ref. 15), multiple solutions were obtained. The "top" branch in our bifurcation diagrams is clearly a flow of Batchelor type while the "bottom" branch is of the Stewartson type. No mathematical connection has so far been observed between these two branches, which appear as disjoint solutions in Figs. 1 and 9, for example. It is possible that, by generalizing this mathematical problem and thus introducing additional physical parameters, perhaps by allowing both disks to rotate or by including a boundary at some finite radius, such a connection between these two solution branches may be found. Nevertheless, this question is not of primary concern in this viscometric investigation. A third "middle" solution branch has also been found, although it is presumably unstable. The stability of these axisymmetric solutions has not been pursued here since that question is not of great importance; rather, the stability of such flows to azimuthal disturbances is of interest, but goes beyond the scope of the present study.

It has been demonstrated numerically here that the deviation of pressure $r^{2} Q(z)$ on the stationary bottom plate depends on the two viscosity parameters $R_{e}$ and $F$ and the rotation rate $\omega$ of the top disk. However, it is also very sensitive to which solution branch is considered. With Reynolds number $R_{e}=1000$, visco-elastic constant $F=10000$, and rotation rate $\omega=0.5$ illustrated in Fig. 5, the ratio of the deviation function $Q(z)$ between the top-branch and bottom-branch solutions is $0.0115 / 10^{-5} \approx 1150$. Thus the Stewartson solution represented by the bottom branch is of little value viscometrically, but the Batchelor-type top-branch solution is of far greater interest. In dimensional variables, if the fluid between the two disks is water, then its kinematic viscosity is approximately $10^{-6} \mathrm{~m}^{2} / \mathrm{s}$, and so the Reynolds number $R_{e}=1000$ assumed in Fig. 5 corresponds to a dimensional separation distance $H \approx 5 \mathrm{~mm}$ between the upper and lower disks, from Eq. (1). Under these circumstances, the rotation rate $\omega=0.5$ corresponds to a dimensional rotation speed $\Omega \approx 22 \mathrm{rad} / \mathrm{s}$ or about 3.5 revolutions/s. Then Fig. 5(b) indicates that the Batchelor-type solution branch produces a dimensionless pressure difference of about $\Delta p=1.5$ across the portion $0<r<11$ of the bottom plate shown, which in dimensional variables represents a pressure difference of approximately $70 \mathrm{~Pa}$ across about $55 \mathrm{~mm}$. This may therefore prove significant in attempts to determine the non-Newtonian coefficient $1 / F$ using pressure measurements on the stationary bottom plate. The much lower Reynolds number $R_{e}=5$ used in Fig. 8 would represent a significantly more viscous fluid than water, if a similar separation distance between the plates is used.

\section{ACKNOWLEDGMENTS}

I am indebted to an anonymous Referee for valuable criticism of an earlier draft of this manuscript.

${ }^{1}$ L. K. Forbes, "On turbulence modelling and the transition from laminar to turbulent flow," ANZIAM J. 56, 28-47 (2014).

${ }^{2}$ L. K. Forbes, "Transition to turbulence from plane Couette flow," ANZIAM J. 57, 89-113 (2015).

${ }^{3}$ L. K. Forbes and M. A. Brideson, "On modelling the transition to turbulence in pipe flow," ANZIAM J. 59, 1-34 (2017).

${ }^{4}$ P. Drazin and W. Reid, Hydrodynamic Stability, 2nd ed. (Cambridge University Press, 2004).

${ }^{5}$ R. Seydel, Practical Bifurcation and Stability Analysis, From Equilibrium to Chaos, 2nd ed. (Springer-Verlag, 1994).

${ }^{6}$ T. Kármán, “Über laminare und turbulente reibung,” J. Appl. Math. Mech./Z. Angew. Math. Mech. 1, 233-252 (1921).

${ }^{7}$ P. J. Zandbergen and D. Dijkstra, "Von Kármán swirling flows," Annu. Rev. Fluid Mech. 19, 465-491 (1987).

${ }^{8}$ R. J. Lingwood and P. H. Alfredsson, "Instabilities of the von Kármán boundary layer," Appl. Mech. Rev. 67, 030803 (2015), 13 pages.

${ }^{9} \mathrm{G}$. Batchelor, "Note on a class of solutions of the Navier-Stokes equations representing steady rotationally-symmetric flow," Q. J. Mech. Appl. Math. 4, 29-41 (1951).

${ }^{10} \mathrm{~K}$. Stewartson, "On the flow between two rotating axial disks," Math. Proc. Cambridge Philos. Soc. 49, 333-341 (1953).

${ }^{11}$ M. M. Holodniok and V. Hlaváček, "Computation of the flow between two rotating coaxial disks: Multiplicity of steady-state solutions,” J. Fluid Mech. 108, 227-240 (1981).

${ }^{12}$ R. Bhatnagar, "Flow of non-Newtonian fluid between two infinite parallel discs for large values of Reynolds number-one rotating and the other at rest," Proc. Indian Acad. Sci. 58, 279-289 (1963).

${ }^{13}$ A. Ahmadpour and K. Sadeghy, "Swirling flow of Bingham fluids above a rotating disk: An exact solution,” J. Non-Newtonian Fluid Mech. 197, 41-47 (2013).

${ }^{14} \mathrm{P}$. T. Griffiths, "Flow of a generalised Newtonian fluid due to a rotating disk," J. Non-Newtonian Fluid Mech. 221, 9-17 (2015).

${ }^{15}$ K. R. Rajagopal, "Flow of viscoelastic fluids between rotating disks," Theor. Comput. Fluid Dyn. 3, 185-206 (1992).

${ }^{16} \mathrm{R}$. Aris, Vectors, Tensors, and the Basic Equations of Fluid Mechanics (Dover, 1962).

${ }^{17}$ J. D. T. L. Harman and N. Richert, Advanced Engineering Mathematics with $M A T L A B, 2$ nd ed. (Brooks and Cole, 2000).

${ }^{18} \mathrm{G}$. Winckel, "Legendre-Gauss quadrature weights and nodes," https://au. mathworks.com/matlabcentral/fileexchange/4540-legendre-gauss-quadratu re-weights-and-nodes, MATLAB File Exchange, 2004. 